\title{
The Effect of Radiotherapy and Chemotherapy on Some Kidney and Liver Functions in Colorectal Cancer Patients
}

\author{
Bashar Abd Alkadhim Naji ${ }^{1}$, Ahmed Sabah Aliand ${ }^{1}$, Ali Slwmee Serhan Al-halaly ${ }^{1}$ \\ ${ }^{1}$ Research, University of Al-Qadisiyah/College of Science/Department of Biology, Iraq
}

\begin{abstract}
The current study was conducted in Al-Diwania Teaching Hospital/Oncology Division and Middle Furat Center for Cancerous Tumors in Al-Najaf, Babil Health Department/Marjan Hospital, Babil Health Department/Imam Al-Sadiq Hospital and Medical City Department/Al-Amal Hospital for the treatment of tumors and continued for the period from 2/10/2019 6/5/2020 60 samples were taken from patients diagnosed with colorectal cancer and 30 samples from non-infected people as a control group, with ages ranging from 33 years to 80 years for both groups. The results showed an increase in the level of Creatinine in the patient group compared to the control group and it was the highest in the radiotherapy group, followed by the chemotherapy group and then the control group. The results showed high significant differences between the three groups $(\mathrm{p}<0.001)$, and there were significant differences between the radiotherapy group. And a chemotherapy group. Through the results, it was found that the average level of Creatinine increased in the radiotherapy and chemotherapy groups compared to the control group. The results showed that the mean of AST was highest in the radiotherapy group, followed by the chemotherapy group and then the control group. The results showed high significant differences between the three groups $(p<0.001)$. Through the results, it was found that there were no significant differences between the radiotherapy group and the chemotherapy group in the average level of the AST enzyme. It was also found that the average level of AST enzyme was higher for the radiotherapy and chemotherapy groups compared to the control group. The results of the current study showed the highest mean ALT enzyme in the radiotherapy group, followed by the chemotherapy group compared to the control group. The results showed high significant differences between the three groups $(p<0.001)$ and significant differences between the radiotherapy group and the chemotherapy group. ALT for the radiotherapy and chemotherapy groups compared to the control group.
\end{abstract}

Keywords: Colorectal cancer, ALT, AST, chemotherapy, radiotherapy.

\section{Introduction}

The kidneys are important vital organs and have many functions, including the production of erythropoietin to stimulate the production of red blood cells, filter metabolites and electrolytes from the blood, and adjust blood pressure through fluid balance ${ }^{(1)}$. Studies have shown the negative effect of chemotherapy agents and their role in the emergence of nephrotoxicity (Nephrotoxicity) and the damage is determined clinically through changes in the rate of glomerular filtration, creatinine clearance, urea nitrogen in the blood and urine excretion, as well as the negative effects of chemotherapy agents that can be radiographed, such as change Cystic, interstitial nephritis, urethral changes, and colitis, etc. ${ }^{(2)}$.Also, radiation therapy causes total body irradiation (TBI) to injure the kidneys with some damage, the most important of which is radiation nephropathy $(\mathrm{RN})^{(3)}$. The incidence of clinical $\mathrm{RN}$ increases with the use of TBI and nephropathy usually develops very slowly over several years as proteinuria, hypertension, and impaired urine concentration ${ }^{(4)}$. Studies have indicated that a dose within the range of 5 to $10 \mathrm{~Gy}$, doses less than 5 Gy will not effectively affect the kidneys, while doses in excess of 10 Gy may cause rapid gastrointestinal death ${ }^{(5)}$. However, given the threshold dose, adequate ionizing radiation affects most or all of the kidney components. The injury to the glomerulus is first with the development of glomerular scars due to thrombotic angiopathy ${ }^{(6)}$. Chemotherapy is one of the main treatments used in treating cancer. Systemic cancer treatment has evolved from traditional toxic 
agents to newer classes of partially targeted therapy. Although these treatments aim to prevent the growth of cancerous tissues, they have harmful effects on normal tissues where many negative effects of chemotherapy can be detected on various organs, including the liver. ${ }^{(7)}$ . Among the most important of these effects is the fatty leaching of the liver tissue and the accumulation of fat cells in the liver cells, which is known as hepatic steatosis or what is known as hepatic steatosis, and steatohepatitis, which is a more severe form of fatty liver disease and is often asymptomatic and can be It appears by elevated ALT and $\mathrm{AST}^{(8)}$. These changes have been linked to chemotherapy agents, Methotrexate 5-fu, Irinotecan, Oxaliplatin ${ }^{(9)}$ as the steatohepatitis (steatohepatitis) associated with chemotherapy can be diffuse or focal and can be seen with an ultrasound scan (US). ${ }^{(10)}$ Hepatic steatosis can also be visualized through computed tomography $(\mathrm{CT})^{(11)}$, as well as magnetic resonance imaging $(\mathrm{MR})^{(12)}$. The detection of lipid changes in a chemotherapy patient is useful because it may prompt changes in treatment, especially in patients with metastatic colorectal cancer, as steatosis may increase the risk of postoperative complications ${ }^{(13)}$.

Several studies have revealed the relationship between acute hepatitis and several chemotherapy treatments ${ }^{(14)}$. Acute hepatitis ranges from asymptomatic inflammation to highly symptomatic inflammation such as nausea, vomiting, poor appetite and jaundice, with an increase in AST and ALT in the majority of patients. Hepatitis improves upon temporary discontinuation of cancer treatment ${ }^{(15)}$. As for the effect of radiation therapy, the liver is exposed indirectly during radiotherapy (RT) to tumors located in the upper abdomen, lower lung, esophagus, entire abdomen or the entire body ${ }^{(16)}$. Despite exposure to a limited area during radiotherapy, normal tissues in that area can receive a high dose of radiation and thus the liver can withstand severe damage such as fibrosis $^{(17)}$. The radiation effects resulting from radiation treatments for cancer patients depend on age and gender ${ }^{(18)}$. It has been observed that radiation damage in cells is like breaking the double strand of DNA and this is at the molecular level and this damage leads to many biochemical reactions, which It subsequently leads to various pathologies such as cirrhosis as a form of acute radiological damage after exposure to high-dose radiation $^{(19)}$. Clinical symptoms known as «classic» radiation induced liver disease (RILD) usually occur. These symptoms occur within four months after exposure to radiation of the liver as the patient suffers from fatigue, weight gain, enlarged liver, and high alkaline phosphatase Which is not compatible with other liver enzymes ${ }^{(20)}$. In contrast to this «classic» RILD, a cancer patient may present with underlying chronic liver disease such as cirrhosis and viral hepatitis with liver function abnormalities such as jaundice within three months of completion of radiotherapy ${ }^{(21)}$.

\section{Materials and Method}

Collecting and testing blood serum: Samples were collected from Al-Diwaniyah Teaching Hospital/ Oncology Department and the Central Euphrates Center for Cancerous Tumors in Najaf, Babil Health Department/Morgan Hospital, Babil Health Department/ Imam Al-Sadiq Hospital and Medical City Department/ Al-Amal Hospital. For the treatment of tumors after the approval of the above-mentioned hospital departments and after taking the verbal consent of the patients and healthy people who were included in the study after clarifying the idea of the research and its purpose, and their data should be used only for the purposes of research and to maintain privacy. $2 \mathrm{ml}$ of the patient $>\mathrm{s}$ blood was withdrawn and it was placed in a centrifuge at $3000 \mathrm{rpm}$ for ten minutes, then the separated serum was taken and transferred to the Eppendorf tube. These samples were kept in continuous freezing temperature $\left(-20^{\circ} \mathrm{C}\right)$. So use it. As for the control samples, 30 samples were taken from healthy people in the same way as patient samples for the purpose of examining some liver and kidney functions.

\section{Examination of Biochemical parameters}

i. Determination of serum creatine levels: Serum creatinine levels were measured using a spinreact Kit.

ii Determination of serum ALT and AST activity (U/L): I used a ready-made test kit, a kit manufactured by the Chinese company CUSABIO, using the color method used by Reitman \& Frankel ${ }^{(22)}$. 


\section{Results}

Table: Biochemical characteristics of colorectal cancer patients and control group

\begin{tabular}{|l|c|c|c|c|}
\hline Characteristic & Control $\boldsymbol{n}=\mathbf{3 0}$ & Radiotherapy $\boldsymbol{n}=\mathbf{3 0}$ & Chemotherapy $\boldsymbol{n}=\mathbf{3 0}$ & $\boldsymbol{P}$-Value \\
\hline \multirow{2}{*}{ Creatinine } & $0.60 \pm 0.05$ & $1.56 \pm 0.08$ & $1.28 \pm 0.06$ & $<0.001$ \\
& $\mathrm{C}$ & $\mathrm{A}$ & $\mathrm{B}$ & HS \\
\hline \multirow{2}{*}{ AST } & $26.04 \pm 0.50$ & $43.75 \pm 0.63$ & $41.14 \pm 1.09$ & $<0.001$ \\
& $\mathrm{~B}$ & $\mathrm{~A}$ & $\mathrm{~A}$ & $\mathrm{HS}$ \\
\hline \multirow{2}{*}{ ALT } & $31.46 \pm 0.29$ & $56.11 \pm 0.54$ & $51.78 \pm 0.32$ & $<0.001$ \\
& $\mathrm{C}$ & $\mathrm{A}$ & $\mathrm{B}$ & $\mathrm{HS}$ \\
\hline
\end{tabular}

The results showed an increase in the level of Creatinine in the patient group compared to the control group and it was highest in the radiotherapy group (1.56 $\pm 0.08)$, followed by the chemotherapy group (1.28 $\pm 0.06)$ and then the control group $(0.60 \pm 0.05)$. The results showed high significant differences between groups. The three were $(\mathrm{p}<0.001)$. There were also significant differences between the radiotherapy group and the chemotherapy group. Through the results, it was found that the average level of Creatinine increased in the radiotherapy and chemotherapy groups compared to the control group.

The results showed that the mean of the highest AST was in the radiotherapy group (43.75 \pm 0.63$)$, followed by the chemotherapy group $(41.14 \pm 1.09)$ and then the control group $(26.04 \pm 0.50)$. The results showed high significant differences between the three groups $(\mathrm{p}<0.001)$. Through the results, it was found that there were no significant differences between the radiotherapy group and the chemotherapy group in the average level of the AST enzyme. It was also found that the average level of the AST enzyme was higher for the radiotherapy and chemotherapy groups compared to the control group.

The results of the current study showed the mean of the higher ALT enzyme in the radiotherapy group (56.11 \pm 0.54 ), followed by the chemotherapy group (51.78 \pm $0.32)$ compared to the control group $(31.46 \pm 0.29)$. The results showed high significant differences between the three groups $(p<0.001)$. Significant levels were found between the radiotherapy group and the chemotherapy group. The mean level of ALT was found to be higher in the radiotherapy and chemotherapy groups compared to the control group.

\section{Discussion}

The percentage of creatinine is high in the serum of cancer patients, and the reason for this increase may be due to kidney injury, as there is a reciprocal relationship between cancer and the kidneys, as chronic kidney disease can increase the risk of cancer, and cancer patients often suffer from renal impairment resulting from associated factors With disease or toxicity of the treatments used by cancer patients, the effect of radiation therapy is more concentrated on cells, being specific to the organ affected by the carcinoid tumor. Therefore, cell lysis in radiotherapy is stronger and faster than chemotherapy ${ }^{(23)}$.Doses of less than 18 Gy of the whole kidney appear to rarely cause severe or long-term renal injury while doses of more than 20 Gy of disease lead to significant nephropathy ${ }^{(24)}$.

(25) found a mean reduction of $8 \%$ in glomerular filtration rate (GFR) for each $100 \mathrm{mg} / \mathrm{m} 2$ dose received from cisplatin treatment. In serum or urine and cisplatin infusion rates. Decreases in glomerular filtration rate and hypomagnesaemia are rare after carboplatin use. Ironically, it is possible that the risk of renal insufficiency and tubopathy is higher with carboplatin/ifosfamide compared to the cisplatin/ifosfamide combination therapy ${ }^{(26)}$. Radiation nephritis or radiation nephropathy develops after a latent period of 3 to 12 months and is manifested in varying degrees of hypertension, renal insufficiency and anemia ${ }^{(27)}$.An elevated liver enzyme level is frequently present in cancer patients but is mostly due to the fact that chemotherapy and radiotherapy damage liver cells ${ }^{(28)}$.

The administration of chemotherapy presents a challenge to the strict regulation and balance of these processes since most drugs tend to be lipophilic, so 
the liver takes them easily. Under the influence of chemotherapy, about $85 \%$ of patients develop liver steatosis and steatohepatitis is the most serious event, especially If it is associated with an increase in bilirubin levels ${ }^{(29)}$.Radiation therapy affects the liver and has negative effects. Treatments cause cirrhosis and venous obstructive liver disease, a feature of patients with radiation-induced liver disease (RILD). Radiation therapy also causes oxidative stress and the production of reactive oxygen species, which lead It leads to the apoptosis of liver cells and acute inflammatory responses and increases the susceptibility of central hepatocytes («centrilobular» hepatocytes «HCs») to the apoptosis of the treatment and ultimately leads to the death and degeneration of hepatocytes. Chemotherapy also causes various tissue changes to the liver, including This fatty degeneration, and steatohepatitis associated with chemotherapy and sinus obstructive syndrome for sinus infection, as well as the treatment regimens and the quality of chemotherapy have a direct and important effect on the severity of the liver injury and this is in accordance with studies ${ }^{(30)}$.

Ethical Clearance: Nil

Source of Funding: Self

Conflict of Interest: Nil

\section{References}

1. Baradaran-Ghahfarokhi, M. «Radiation-induced kidney injury». Journal of renal injury prevention. (2012), 1(2), 49.

2. Ngo, D., Jia, J. B., Green, C. S., Gulati, A. T., \& Lall, C. «Cancer therapy related complications in the liver», pancreas, and biliary system: an imaging perspective. Insights into imaging.(2015), 6(6), 665-677.

3. Cohen, E. P., \& Robbins, M. E.» Radiation nephropathy». In Seminars in nephrology. WB Saunders. (2003), September,Vol. 23, No. 5, pp. 486-499.

4. Pan, C. C., Kavanagh, B. D., Dawson, L. A., Li, X. A., Das, S. K., Miften, M., \& Ten Haken, R. K.» Radiation-associated liver injury». International Journal of Radiation Oncology* Biology* Physics .(2010)., 76(3), S94-S100.

5. Sohn, W., Clayman, R. V., Lee, J. Y., Cohen, A., \& Mucksavage, P..»Low-dose and standard computed tomography scans yield equivalent stone
measurements».Urology. (2013), 81(2), 231-235.

6. Baradaran-Ghahfarokhi, M. «Radiation-induced kidney injury». Journal of renal injury prevention .(2012), 1(2), 49.

7. Ngo, D., Jia, J. B., Green, C. S., Gulati, A. T., \& Lall, C. «Cancer therapy related complications in the liver», pancreas, and biliary system: an imaging perspective. Insights into imaging .(2015), 6(6), 665-677.

8. Robinson, S. M., Scott, J., Manas, D. M., \& White, S. A. «The assessment and management of chemotherapy associated liver injury». Hepatic Surgery. Intech Open, Rijeka(2013), 397-421.

9. King, P. D., \& Perry, M. C.» Hepatotoxicity of chemotherapy». The oncologist .(2001), 6(2), 162176.

10. Robinson, P. J. «The effects of cancer chemotherapy on liver imaging». European radiology (2009), 19(7), 1752-1762.

11. Sharma, A., Houshyar, R., Bhosale, P., Choi, J. I., Gulati, R., \& Lall, C.»Chemotherapy induced liver abnormalities»: an imaging perspective. Clinical and molecular hepatology .(2014), 20(3), 317.

12. Décarie, P. O., Lepanto, L., Billiard, J. S., Olivié, D., Murphy-Lavallée, J., Kauffmann, C., \& Tang, «.A. Fatty liver deposition and sparing»: a pictorial review. Insights into imaging .(2011), 2(5), 533538.

13. Torrisi, J. M., Schwartz, L. H., Gollub, M. J., Ginsberg, M. S., Bosl, G. J., \& Hricak, H.» CT findings of chemotherapy-induced toxicity»: what radiologists need to know about the clinical and radiologic manifestations of chemotherapy toxicity. Radiology .(2011), 258(1), 41-56.

14. Maor, Y., \& Malnick, S.» Liver injury induced by anticancer chemotherapy and radiation therapy». International journal of hepatology .(2013), 2013, 815105

15. King, P. D., \& Perry, M. C. «Hepatotoxicity of chemotherapy». The oncologist .(2001), 6(2), 162176.

16. Tong, M. J., Pan, C. Q., Hann, H. W., Kowdley, K. V., Han, S. H. B., Min, A. D., \& Leduc, T. S.» The management of chronic hepatitis B in Asian Americans». Digestive diseases and sciences .(2011), 56(11), 3143.

17. Xu, Z. Y., Liang, S. X., Zhu, J., Zhu, X. D., Zhao, 
J. D., Lu, H. J., ... \& Jiang, G. L.» Prediction of radiation-induced liver disease by Lyman normaltissue complication probability model in threedimensional conformal radiation therapy for primary liver carcinoma». International Journal of Radiation Oncology* Biology* Physics .(2006), 65(1), 189-195.

18. Guha, C., \& Kavanagh, B. D.» Hepatic radiation toxicity»: avoidance and amelioration. In Seminars in radiation oncology. WB Saunders. (2011), October,Vol. 21, No. 4, pp. 256-263)

19. Liang, S. X., Zhu, X. D., Xu, Z. Y., Zhu, J., Zhao, J. D., Lu, H. J., ... \& Jiang, G. L.» Radiation-induced liver disease in three-dimensional conformal radiation therapy for primary liver carcinoma»: the risk factors and hepatic radiation tolerance. International Journal of Radiation Oncology Biology Physics. (2006), 65(2), 426-434.

20. Reitman, S., \& Frankel, S. «A colorimetric method for the determination of serum glutamic oxalacetic and glutamic pyruvic transaminases». American journal of clinical pathology. (1957), 28(1), 56-63.
21. Shahinian, V. B., Bahl, A., Niepel, D., \& Lorusso, $\mathrm{V} . »$ Considering renal risk while managing cancer». Cancer management and research .(2017), 9, 167178.

22. Jones, D. P., Spunt, S. L., Green, D., Springate, J. E., \& Children〉s Oncology Group «.Renal late effects in patients treated for cancer in childhood»: a report from the Children $>$ S Oncology Group. Pediatric blood \& cancer .(2008), 51(6), 724-731.

23. Cohen, E. P., \& Robbins, M. E.» Radiation nephropathy». In Seminars in nephrology. WB Saunders. (2003), September, (Vol. 23, No. 5, pp. 486-499.

24. Guha, C., \& Kavanagh, B. D.» Hepatic radiation toxicity»: avoidance and amelioration. In Seminars in radiation oncology WB Saunders. (2011), October,Vol. 21, No. 4, pp. 256-263.

25. Ramadori, G., \& Cameron, S.» Effects of systemic chemotherapy on the liver». Annals of Hepatology .(2010), 9(2), 133-143.

26. Maor, Y., \& Malnick, S.» Liver injury induced by anticancer chemotherapy and radiation therapy». International journal of hepatology .(2013), 2013, 815105 . 